\begin{tabular}{|c|l|}
\hline Title & $\begin{array}{l}\text { Magnetic domain structure and domain wall analy sis of ferromagnetic MnA s nanodisks selectively-grown on Si (111) } \\
\text { substrates for spintronic applications }\end{array}$ \\
\hline Author(s) & Horiguchi, Ryoma; Hara, Shinjiro; Iida, Masaya \\
\hline Citation & $\begin{array}{l}\text { Journal of A pplied Physics, 124(15), 153905 } \\
\text { https://doi.org/10.1063/1.5045241 }\end{array}$ \\
\hline Issue Date & 2018-10-21 \\
\hline Doc URL & http://hdl.handle.net/2115/75793 \\
\hline Rights & $\begin{array}{l}\text { The following article appeared in Journal of A pplied Physics 124, Issue 15, 153905 (2018) and may be found at } \\
\text { https://doi.org/10.1063/1.5045241. }\end{array}$ \\
\hline Type & article \\
\hline File Information & \begin{tabular}{l}
$1.5045241 . p d f$ \\
\hline
\end{tabular} \\
\hline
\end{tabular}

Instructions for use 


\section{Magnetic domain structure and domain wall analysis of ferromagnetic MnAs nanodisks selectively-grown on Si (111) substrates for spintronic applications}

Cite as: J. Appl. Phys. 124, 153905 (2018); https://doi.org/10.1063/1.5045241

Submitted: 19 June 2018 . Accepted: 27 September 2018 . Published Online: 18 October 2018

Ryoma Horiguchi, Shinjiro Hara, and Masaya lida
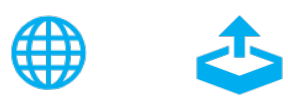

\section{ARTICLES YOU MAY BE INTERESTED IN}

Phase separation and effect of strain on magnetic properties of $\mathrm{Mn}_{3} \mathrm{Ga}_{1-\chi} \mathrm{Sn}{ }_{\chi} \mathrm{C}$ Journal of Applied Physics 124, 153902 (2018); https://doi.org/10.1063/1.5050655

Tuning magnetic anisotropy of epitaxial $\mathrm{Ag} / \mathrm{Fe} / \mathrm{Fe}_{0.5} \mathrm{C}_{0} 0.5 / \mathrm{MgO}(001)$ films Journal of Applied Physics 124, 153904 (2018); https://doi.org/10.1063/1.5052297

The effective point charge of probe tip in piezoresponse force microscopy Journal of Applied Physics 124, 154106 (2018); https://doi.org/10.1063/1.5047006

\section{Applied Physics Reviews} Now accepting original research 


\title{
Magnetic domain structure and domain wall analysis of ferromagnetic MnAs nanodisks selectively-grown on Si (111) substrates for spintronic applications
}

\author{
Ryoma Horiguchi, ${ }^{\text {a) }}$ Shinjiro Hara, ${ }^{\text {b) }}$ and Masaya lida \\ Research Center for Integrated Quantum Electronics, Hokkaido University, Sapporo 060-0813, Japan
}

(Received 19 June 2018; accepted 27 September 2018; published online 18 October 2018)

\begin{abstract}
We report on the experimental and analytic results on magnetic domain and domain wall structures of MnAs nanodisks on AlGaAs nanopillar buffers selectively grown on Si (111) substrates partially covered with dielectric $\mathrm{SiO}_{2}$ thin film mask patterns using selective-area metal-organic vapor phase epitaxy. The results on the size dependence of the magnetic domain structure in MnAs nanodisks investigated by magnetic force microscopy show that a single domain is predominant in the MnAs nanodisks with an area of approximately $3 \times 10^{4} \mathrm{~nm}^{2}$ or less. It is also indicated that in the nanodisks with an area of approximately $6 \times 10^{4} \mathrm{~nm}^{2}$ or more, multiple domains, in particular, two magnetic domain structures with a $180^{\circ}$ domain wall, are predominant. In addition, in the case of nanodisks with multiple domains, not only Néel walls but also Bloch walls are possibly formed, according to the detailed analyses of the magnetic force microscope images obtained. These results suggest that the magnetic domains and domain walls can be tuned by the control of the MnAs nanodisk size making them interesting nanostructures for spintronic applications. Published by AIP Publishing. https://doi.org/10.1063/1.5045241
\end{abstract}

\section{INTRODUCTION}

In the field of semiconductor electronics, device performance has been improved by the miniaturization of the devices. However, because of the technological limitation on fabrication size of devices, the nanodevices based on new functionalities and operation principles have been intensively investigated. Among new nanotechnologies, the research field of nanospintronics in particular, in which two degrees of freedom of the electrons, i.e., a charge and spins, are utilized in devices, has attracted considerable attention. ${ }^{1,2}$ Magnetic tunnel junctions (MTJs) are highly expected to realize a nonvolatile memory among such nanospintronic devices because large magnetoresistance effects have been demonstrated in the MTJs, in which the spin states of electrons are preserved without any energy consumption, in combination with semiconducting materials. $^{3-5}$ In addition, racetrack-type memory cell arrays integrated on Si substrates with MTJ read elements and complementary metal-oxide semiconductor field-effect transistors have been intensively investigated. ${ }^{6,7}$ In magnetic memories, in general, the thermal stability factor, i.e., $K V /$ $k_{B} T$, must exceed 60 at least to ensure that the magnetization direction of the magnetic material remains stable for archiving and data retention in 10 years, where $K, V, k_{B}$, and $T$ are the magnetic anisotropy constant, the volume of magnetic materials, Boltzmann constant, and temperature, respectively. ${ }^{8,9}$ To miniaturize magnetic memories, a high magnetic anisotropy constant, $K$, is required. As MnAs bulk crystals show ferromagnetism at room temperature and have a relatively high uniaxial magnetic anisotropy energy, ${ }^{10,11}$ intensive studies on the MTJ structures using MnAs have

\footnotetext{
${ }^{a)}$ E-mail: horiguchi@ rciqe.hokudai.ac.jp

b) Author to whom correspondence should be addressed: hara@ rciqe.hokudai.ac.jp
}

been performed. ${ }^{4,5}$ In particular, a huge magnetoresistance up to $100000 \%$ has been reported in MTJs using granular GaAs:MnAs hybrid nanomaterial system consisting of ferromagnetic MnAs nanoclusters embedded in semiconducting GaAs layers. ${ }^{5}$ Such MTJ structures have been mainly fabricated by conventional top-down-type microfabrication technologies combined with low-temperature molecular beam epitaxy of thin films. However, the performance of devices fabricated by such a conventional method possibly deteriorates owing to process-induced damages and a relatively poor size-uniformity, in particular, on the way toward a further miniaturization of devices on the nanometer scale.

To solve such potential problems, we have developed a bottom-up-type fabrication method, which is based on selective-area metal-organic vapor phase epitaxy (SA-MOVPE), of ferromagnetic MnAs nanostructures on semiconducting GaAs (111)B and Si (111) substrates. ${ }^{12-17}$ This technique enables us to control the size, density, position, and shape of nanostructures without any process-induced damage and contamination for the creation of nanospintronic devices, such as magnetic memories using MTJs, with ferromagnetic MnAs nanostructures. The MTJ structures are fabricated by conventional lithography processes after the deposition of oxide interlayers (e.g., $\mathrm{MgO}, \mathrm{Al}_{2} \mathrm{O}_{3}$ ) and ferromagnetic thin films on the MnAs nanodisks (NDs) for the vertical-type MTJs. In addition, for the lateral-type MTJs, we believe that the use of elongated MnAs ND composites is promising, as demonstrated on GaAs (111)B substrates in our previous study. ${ }^{16}$ In general, the magnetic domain structure depends on the size and shape of the magnetic nanomaterial. In this paper, we therefore characterize and analyze in detail the magnetic domains and domain walls in magnetic MnAs ND structures selectively grown on Si (111) substrates. ${ }^{17}$ Using dielectric $\mathrm{SiO}_{2}$ thin film mask patterns as the growth inhibiting layers for SA-MOVPE allows us to 
control the magnetic domain structure by controlling the MnAs ND size.

\section{EXPERIMENTAL DETAILS}

We have investigated the SA-MOVPE growth and characterized the structural properties of magnetic MnAs ND structures after the growth of AlGaAs nanopillar (NP) buffers on $\mathrm{Si}$ (111) substrates using multiple types of mask materials and designs. The fabrication procedure for the NDs used in the current work is described and explained in detail elsewhere. ${ }^{17}$ In brief, we fabricated the MnAs NDs after the growth of AlGaAs NP buffers on $\mathrm{Si}$ (111) substrates covered with a 30-nm-thick $\mathrm{SiO}_{2}$ mask pattern, in which the dielectric $\mathrm{SiO}_{2}$ thin film is partly removed to prepare periodically arranged circular openings within $100 \times 100 \mu \mathrm{m}^{2}$ square regions. These periodically arranged circular openings in the $\mathrm{SiO}_{2}$ thin films act as a window for the selective-area growth of both the AlGaAs NP buffers and the MnAs NDs. ${ }^{17}$ For the size estimation of the MnAs NDs, backscattered electron (BSE) observations using scanning electron microscopy (SU8010 system, Hitachi) were carried out. The BSE imaging is sensitive to solid compositions of the observed material because the BSE emission increases with increasing mean atomic number of the material. ${ }^{18}$ The acceleration voltage dependence of the BSE images was analyzed to estimate roughly the thickness of the MnAs NDs on the AlGaAs NP buffers. For the magnetic domain characterization of the NDs, magnetic force microscopy (MFM) combined with atomic force microscopy (AFM) in a Nanoscope IIIa system (Digital Instruments) were performed at room temperature using a high-resolution-type MFM tip of $\mathrm{Si}$ coated with a $\mathrm{Co} / \mathrm{Cr}$ alloy (Bruker MESP-HR10). To investigate the magnetic switching behavior in the NDs, the magnetic domain structure was investigated before and after applying an external magnetic field, $\boldsymbol{B}$, up to 1000 Gauss (G) using a conventional stand-alone electromagnet. It should be noted that during the MFM measurements, no magnetic field was applied. The coercive force, or coercivity, of the MFM tips used in the current work is $950 \mathrm{Oe}$ according to the specification sheet published by Bruker AXS.

\section{RESULTS AND DISCUSSION}

\section{A. Structural characterization of MnAs NDs}

For the size estimation of the MnAs NDs, first, we carried out BSE observations. Figures 1(a) and 1(b) show the dependence of top view images of MnAs NDs grown on AlGaAs NP buffers on the acceleration voltage for the BSE observations. In the case of an acceleration voltage of $0.5 \mathrm{kV}$, we observe a relatively bright image contrast in the ND marked by "A" (hereafter, it is referred to as ND-A) and a darker image contrast in the ND marked by "B" (ND-B) than the image contrast in ND-A, as shown in Fig. 1(a). BSE images are highly sensitive to the solid composition of a material since the BSE emission increases with increasing the mean atomic number of the material. ${ }^{18}$ Since the mean atomic number of AlGaAs is larger than that of MnAs, BSE images of the AlGaAs NP buffers should be brighter than those of the MnAs NDs. Therefore, we conclude that the

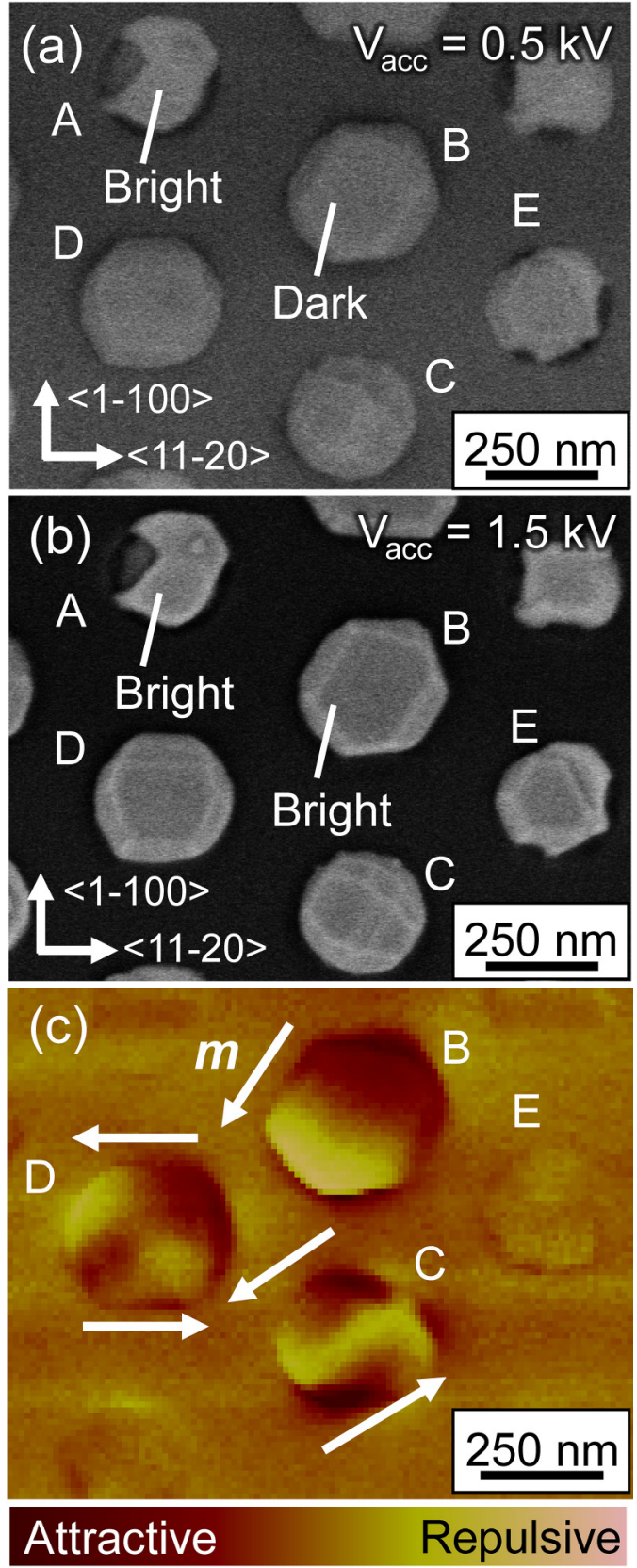

FIG. 1. Acceleration voltage dependence of BSE images of MnAs NDs on AlGaAs NP buffers: (a) 0.5 and (b) $1.5 \mathrm{kV}$. In (a), a relatively bright image contrast of ND-A and an image contrast of ND-B darker than the one of ND-A are observed. In (b), almost no significant difference in the image contrasts between ND-A and ND-B is observed. (c) MFM image of the corresponding MnAs NDs and AlGaAs NP buffers. The MFM observations were carried out at room temperature without any application of an external magnetic field. Most of the MnAs NDs show a spontaneous magnetization. A single magnetic domain is observed in ND-B, and multiple magnetic domains are observed in ND-C and ND-D. The white arrows in (c) indicate the directions of the magnetic moments $(\boldsymbol{m})$ in the NDs. No well-defined contrast is observed in the MFM image of ND-E.

dark and bright image contrasts are due to MnAs NDs and AlGaAs NP buffers grown under the MnAs NDs, respectively. Increasing the acceleration voltage for the BSE images to $1.5 \mathrm{kV}$, almost no significant difference in the image contrasts between ND-A and ND-B are observed, i.e., the image contrast of ND-B became almost the same as that of ND-A, as shown in Fig. 1(b). The penetration length of electrons 
depends on an acceleration voltage of electrons in scanning electron microscopy. Therefore, in the case of an acceleration voltage of $1.5 \mathrm{kV}$, the bright image contrasts observed in most of the positions, where the MnAs NDs are located, are mainly due to the AlGaAs NP buffers grown under the MnAs NDs, as shown in Figs. 1(a) and 1(b). (However, no MnAs ND but only the AlGaAs NP buffer was grown actually in "ND-A," finally judging from the BSE and MFM analyses in Fig. 1.) The penetration length of electrons into MnAs NDs was calculated to be approximately $14 \mathrm{~nm}$ at $1.0 \mathrm{kV}$ and $27 \mathrm{~nm}$ at $1.5 \mathrm{kV}$ using the equation given in Ref. 19 . Therefore, we roughly estimate a MnAs ND thickness of $20 \mathrm{~nm}$ in the observed NDs. In our previous study ${ }^{17}$ the thickness of MnAs NDs grown on Si (111) substrates covered with SiON mask patterns under the same growth conditions was estimated to be approximately $10 \mathrm{~nm}$ by transmission electron microscopy (TEM). The thickness estimated from the TEM images for a comparable structure is smaller than that roughly estimated by the BSE imaging, but of the same order of magnitude. It is difficult to estimate the thickness of MnAs NDs by AFM since the measured thickness is the total thickness of a MnAs ND and an AlGaAs NP buffer. However, the mean thickness of some MnAs NDs partially grown on AlGaAs NP buffers, which were unintentionally and occasionally observed, was estimated to be $22.2 \mathrm{~nm}$ by AFM. This mean thickness estimated by AFM is in good agreement with the above-mentioned thickness estimated from the BSE images, i.e., approximately $20 \mathrm{~nm}$. From electron beam diffraction measurements by TEM, we conclude that the crystallographic structure of all MnAs NDs observed in the current work is a hexagonal NiAs-type structure and that the $<0001>$ direction of hexagonal NiAs-type MnAs is parallel to both the $<111>\mathrm{B}$ direction of the zinc-blende-type AlGaAs NP buffers and the <111> direction of the diamond-type Si substrates (not shown here).

\section{B. Magnetic domain and domain wall characterizations of MnAs NDs}

First of all, we conducted MFM observations at room temperature without the application of an external magnetic field to characterize the magnetic domain structure of the MnAs NDs. Figure 1(c) shows an MFM image of the MnAs NDs shown in Figs. 1(a) and 1(b). Well-defined image contrasts are observed for most of the NDs. Therefore, the MnAs NDs in Fig. 1 show a spontaneous magnetization at room temperature. A single magnetic domain is markedly confirmed in ND-B in Fig. 1(c). In addition, multiple magnetic domains are observed in the NDs marked by "C" (ND-C) and "D" (ND-D). The white arrows in the image represent the directions of the magnetic moment $(\boldsymbol{m})$ in the MnAs NDs. On the other hand, no welldefined contrast is observed in the MFM images for some of the NDs, although relatively dark contrasts are confirmed in the BSE images owing to the MnAs NDs, e.g., as in the case of the ND marked by "E" (ND-E) in Fig. 1. A MnAs bulk crystal shows a first-order structural phase transition between the paramagnetic orthorhombic $\beta$-phase ( $\beta$-MnAs, MnP-type) and the ferromagnetic hexagonal $\alpha$-phase ( $\alpha$-MnAs, NiAs-type) at temperatures of 317 to $319 \mathrm{~K}^{20,21}$ In the case of heteroepitaxial
MnAs thin films, it was reported that a phase coexistence of $\alpha$-MnAs and $\beta$-MnAs was observed in MnAs thin films grown on GaAs $(001)^{22,23}$ and (111)B substrates. ${ }^{24}$ On GaAs (001) surfaces, MnAs thin films showed a strain-stabilized coexistence of the $\alpha$-MnAs and $\beta$-MnAs phase resulting in a stripe structure, ${ }^{22,23}$ whose periodicity depended on the thickness of the MnAs thin films. ${ }^{25}$ The results in Fig. 1(c), therefore, suggest that the NDs are formed either as a ferromagnetic $\alpha$-MnAs phase or a paramagnetic $\beta$-MnAs phase. Then, in the case of ND-E, for example, no well-defined contrast, i.e., no marked magnetic response, in the MFM image may be due to the $\beta$-MnAs phase. (At least, the possibility of the existence of paramagnetic $\beta$-MnAs NDs has thus far not yet been excluded from the MFM analysis in the current work, although the crystallographic structure of all the other MnAs NDs observed by TEM was of hexagonal NiAs-type, i.e., a ferromagnetic $\alpha-M n A s$ phase.) Figure 2 shows the size dependence of the
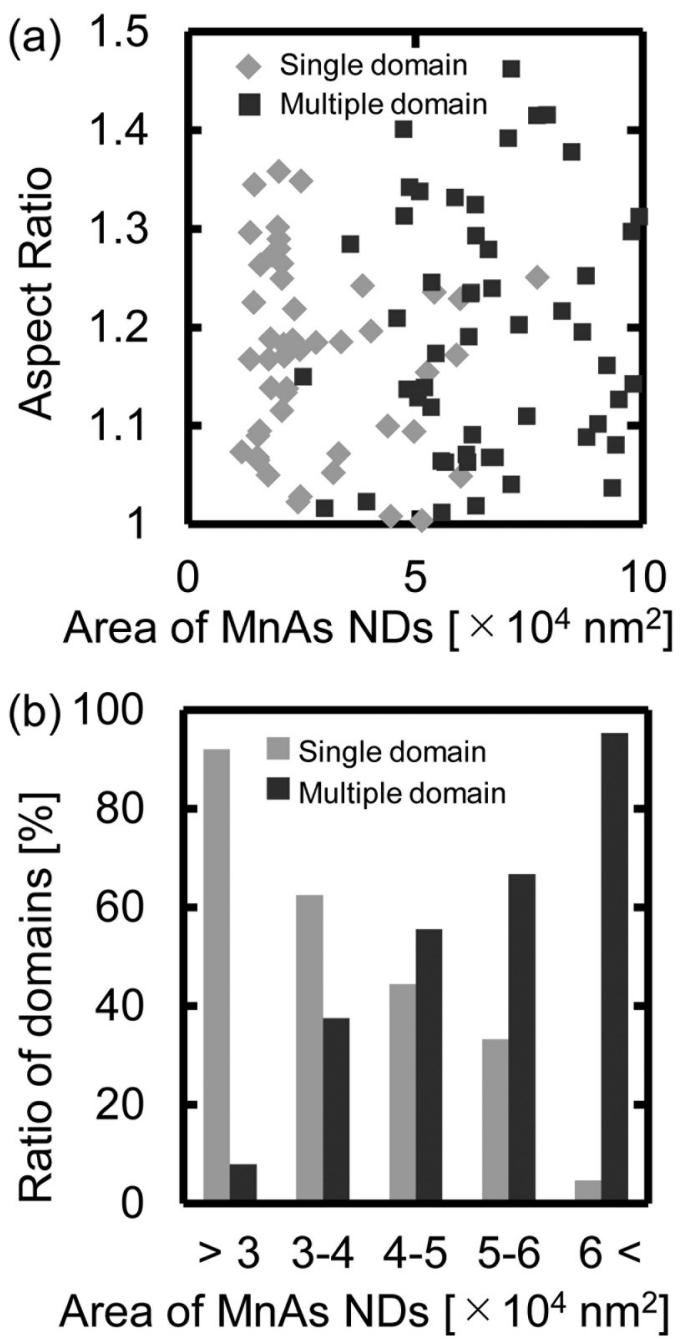

FIG. 2. Size dependence of the magnetic domain structure of MnAs NDs on AlGaAs NP buffers. (a) The two-dimensional distributions of single and multiple magnetic domains as a function of the area and the aspect ratio of the MnAs NDs. The aspect ratio is defined as the ratio of the longest length of the $<11-20>$ direction divided by the shortest length of the $<1-100>$ direction of the MnAs NDs. (b) The ratio of the domain structure as a function of area of the MnAs NDs. The number of collected data is 100. Single magnetic domain structures are mainly observed in NDs with an area of $3 \times 10^{4}$ $\mathrm{nm}^{2}$ or less, and multiple magnetic domain structures are observed in NDs with an area of $6 \times 10^{4} \mathrm{~nm}^{2}$ or more. 
magnetic domain structures in our MnAs NDs. The aspect ratios and the areas of the MnAs NDs were estimated from the top views of the BSE images. The aspect ratio is defined as the ratio of the length along the $<11-20>$ direction divided by that along the $<1-100\rangle$ direction of the MnAs NDs. The number of analyzed NDs is 100 in Fig. 2(b). Single and multiple magnetic domains are observed in most of the MnAs NDs with an area of $3 \times 10^{4} \mathrm{~nm}^{2}$ or less and $6 \times 10^{4} \mathrm{~nm}^{2}$ or more, respectively. In the small NDs, the domain wall energy generated by dividing a single domain into two magnetic domains is higher than the magneto-static energy in a single domain state. On the other hand, in the NDs with a comparatively large size, this domain wall energy is lower than the magneto-static energy in a single domain state. Therefore, it is reasonable that single and multiple domain structures are formed in the small and large NDs, respectively. A $180^{\circ}$ domain wall is mainly confirmed for the multiple domain structures, such as ND-C and ND-D shown in the schematic illustrations of Figs. 3(a) and 3(b). In the part corresponding to the domain wall of ND-C illustrated in Fig. 3(a), strong repulsive forces between the magnetic moment at the possible domain wall center and the magnetization of the MFM tip, i.e., large effective stray magnetic fields in the direction perpendicular to the substrate plane, are observed, as shown in Fig. 3(c). On the other hand, in the case of ND-D illustrated in Fig. 3(b), the observed repulsive forces at the possible domain wall center were not as strong as those in the case of ND-C shown in Fig. 3(d). In MnAs thin films with Bloch walls, the image contrast pattern similar to the one obtained in ND-C was observed by other research groups. ${ }^{26}$ Therefore, it is highly possible that ND-C and ND-D exhibit Bloch and Néel walls, respectively. Subsequently, we estimated the domain wall widths of ND-C and ND-D from the contrast patterns, or contrast distributions, of the MFM images shown in Fig. 1. The estimation of the domain wall widths from the MFM image contrast has been reported by several other groups. ${ }^{27-29}$ In the papers published, in general including the articles (Refs. 27-29), the domain wall widths, i.e., Bloch or Néel wall widths, were estimated as the widths between the maximum and minimum peaks of the MFM image contrast generated by the stray magnetic fields from the magnetizations in two magnetic domains. Therefore, it seemed that these estimated widths were overestimated to some extent because the actual domain wall width should not be the distance between two magnetic domain centers, i.e., not between the maximum and minimum peaks of the MFM image contrast. In addition, the stray magnetic fields away from the sample surfaces were detected and imaged by the MFM tip as the tip was somewhat lifted up from the sample surfaces during the MFM scans. However, the experimental domain wall widths estimated from the MFM observation results were well-described by the simulated domain wall widths, as discussed in Ref. 27. Therefore, the overestimation of a domain wall width does not seem to be a major problem for magnetic structures on the micrometer scale $^{27,28}$ since the tip height during the MFM observations is probably negligible compared to the size of the magnetic microstructure. However, we believe that the overestimation of the domain wall width in MFM images may become a major issue to be taken care of for structures on the nanometer scale. In the current work, the stray magnetic fields from the MnAs NDs were detected at a tip height of $30 \mathrm{~nm}$ during all the MFM observations. The radius of the NDs estimated from the MFM images is approximately $7 \%$ to $10 \%$ larger than that estimated from the AFM images. When estimating the Bloch wall width, $W_{\text {Bloch }}$, in ND-C, we assumed that the perpendicular component of the magnetic moments changes according to a sine curve along the Bloch wall. Thus, the intensity gradient of
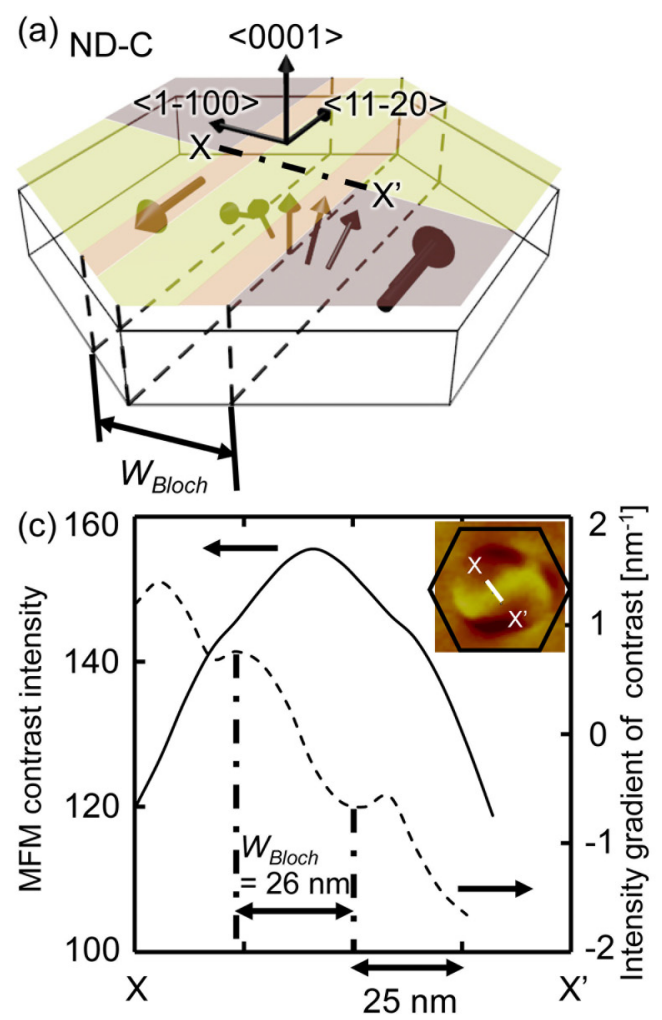
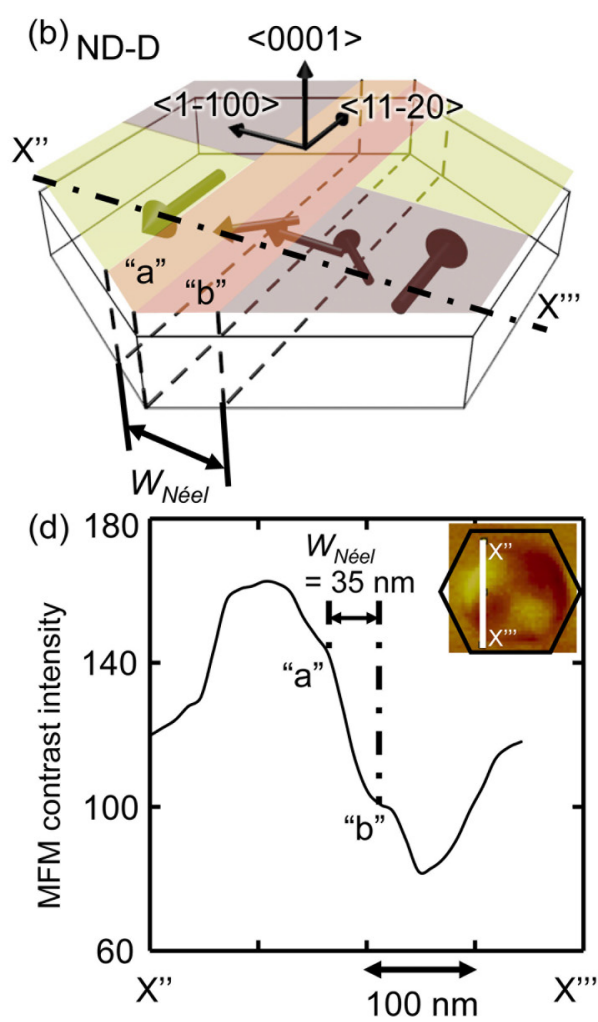

FIG. 3. Schematic illustrations of the magnetic domain structures and the domain walls assumed by the contrasts of the MFM images in (a) ND-D and (b) ND-C. Bloch and Néel wall widths are estimated to be approximately 26 $\mathrm{nm}$ for ND-D and $35 \mathrm{~nm}$ for ND-C, respectively, by the image contrasts and the intensity gradient of the contrasts in the MFM images of (c) ND-D and (d) ND-C. 
the image contrasts should change according to a sine curve along the Bloch wall, as observed in the calculated intensity gradient indicated by the dashed line in Fig. 3(c). From this, a width of approximately $26 \mathrm{~nm}$ is estimated as the Bloch wall width, i.e., $W_{\text {Bloch }}=26 \mathrm{~nm}$. Subsequently, we also estimated the width of the Néel wall, $W_{\text {Néel }}$, in ND-D. Two kinks marked by " $a$ " and " $b$ " are observed in the intensity distribution curve of image contrasts, as shown in Fig. 3(d). These two kinks, a and $b$, can be attributed to additional repulsive and attractive forces detected by the MFM tip, which are generated by the sum of all the effective magnetic moments during the in-plane rotation in the Néel wall. Therefore, we determined the width between the two kinks, resulting in an estimated Néel wall width of approximately $35 \mathrm{~nm}$, i.e., $W_{\text {Néel }}=35 \mathrm{~nm}$. When neglecting the influence of the stray field energy density, the theoretical wall widths for Bloch and Néel walls in MnAs can be calculated using Eqs. (1) and (2), given in Ref. 30,

$$
\begin{aligned}
& W_{\text {Bloch }}=\sqrt{2} \pi \sqrt{\frac{A}{K_{<0001>}}}, \\
& W_{\text {Néel }}=\sqrt{2} \pi \sqrt{\frac{A}{K_{<1-100>}}},
\end{aligned}
$$

where $A$ is the exchange stiffness constant, and $K_{<0001>}$ and $K_{<1-100>}$ are the anisotropy energies in the $<0001>$ and $<1-100>$ directions of hexagonal NiAs-type MnAs, respectively. With the reported values, $A=1.0 \times 10^{-6} \mathrm{erg} / \mathrm{cm},{ }^{23}$ $K_{<0001>}=5.2 \times 10^{6} \mathrm{erg} / \mathrm{cm}^{3},{ }^{10}$ and $K_{<1-100>}=2.2 \times 10^{6} \mathrm{erg} /$ $\mathrm{cm}^{3}{ }^{10}$ the theoretical domain wall widths are calculated to be approximately $19 \mathrm{~nm}$ for the Bloch wall and $30 \mathrm{~nm}$ for the Néel wall. Thus, the calculated domain wall widths are to some extent smaller than those estimated by the contrasts in MFM images, but of the same order of magnitude.

In general, the formation of a Néel wall is preferred in thin films since the magneto-static energy increases with decreasing film thickness for magnetic moments oriented along the direction perpendicular to the plane surface. To discuss the probability for the formation of Bloch and Néel walls in the NDs, we calculated the domain wall energies. Here, we assumed that the shape of the NDs was discoid for simplification. The thickness dependence of the Bloch and Néel wall energies calculated in Ni-Fe thin films was reported by other research groups. ${ }^{31-33}$ As reported by Middelhoek, ${ }^{30}$ the energies per unit areas for a Bloch wall, $\gamma_{\text {Bloch }}$, and a Néel wall, $\gamma_{\text {Néel }}$, were obtained by adding the exchange, the anisotropy, and the stray field energy densities, i.e.,

$\gamma_{\text {Bloch }}=A\left(\frac{\pi}{W_{\text {Bloch }}}\right)^{2} W_{\text {Bloch }}+\frac{K_{<0001>}}{2} W_{\text {Bloch }}+\frac{\pi W_{\text {Bloch }}{ }^{2} M_{s}{ }^{2}}{W_{\text {Bloch }}+D}$,

$\gamma_{\text {Néel }}=A\left(\frac{\pi}{W_{\text {Néel }}}\right)^{2} W_{\text {Néel }}+\frac{K_{<1-100>}}{2} W_{\text {Neel }}+\frac{\pi W_{\text {Neel }} D M_{s}^{2}}{W_{\text {Neel }}+D}$,

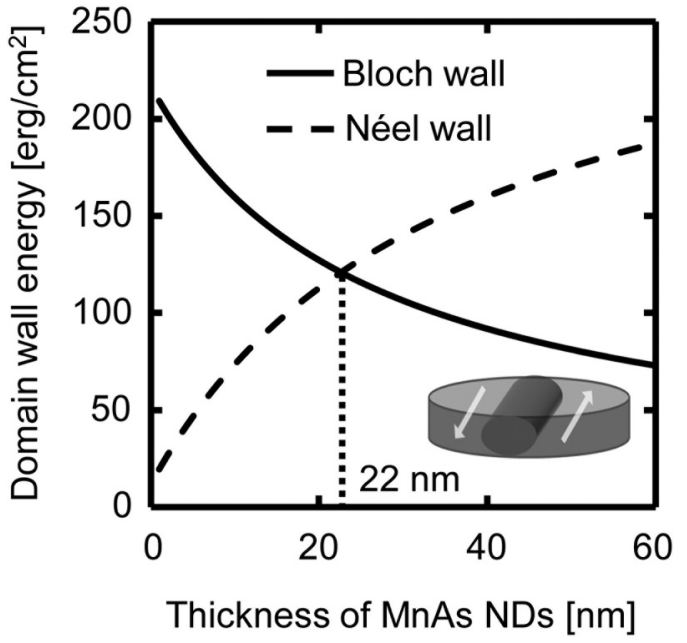

FIG. 4. Calculated wall energy density per unit area of the domain walls as a function of the MnAs thickness. The Bloch wall energy is equal to the Néel wall energy at a ND thickness of approximately $22 \mathrm{~nm}$, which is in good agreement with the thickness of $20 \mathrm{~nm}$ roughly estimated from the acceleration voltage dependence of the BSE images of the MnAs NDs.

where $M_{s}$ is the saturation magnetization, and $D$ is the thickness of the thin films, i.e., MnAs NDs in the current work. Using Eqs. (3) and (4), the energies of the Bloch and Néel walls were calculated, and the results are plotted in Fig. 4. Here, $M_{s}=400 \mathrm{emu} / \mathrm{m}^{3}$ (Ref. 23) was used to calculate the domain wall energies. The calculations reveal that the energy of a Bloch wall is equal to that of a Néel wall when the thickness of MnAs NDs is approximately $22 \mathrm{~nm}$, which is in good agreement with the thickness estimated from the BSE images, i.e., approximately $20 \mathrm{~nm}$. Therefore, we concluded that it is reasonable to observe not only MnAs NDs with Néel walls but also with Bloch walls in the current work. These results suggest that the magnetic domains and the type of domain walls can be tuned by the size of the MnAs NDs.

To characterize the applied external magnetic field dependence of the magnetic domain structure, we applied an external magnetic field, $\boldsymbol{B}$, to the NDs. Figure 5(a) shows a typical BSE image of the ND marked by "F" (ND-F). First, in the case of the as-grown ND-F, i.e., no application of $\boldsymbol{B}$ to ND-F, a single magnetic domain is markedly observed with a spontaneous magnetization, as shown in Fig. 5(b). The magnetization direction is indicated by a thin white arrow in Fig. 5(b). After the application of a magnetic field $\boldsymbol{B}=500 \mathrm{G}$ in the opposite direction to the magnetization direction of $\mathrm{ND}-\mathrm{F}$, the change from single to multiple magnetic domain structures, i.e., a domain wall nucleation, was observed, as shown in Fig. 5(c). In the case of Fe-Nd-B magnets, it was reported that the nucleation of domain walls occurs at the defects on the particle surfaces. ${ }^{34}$ In our previous study, ${ }^{17}$ the dislocations, whose mean period was estimated to be approximately $3.6 \mathrm{~nm}$, were observed only in one or two monolayers of the MnAs layers in the NDs at the interfaces between the MnAs and the AlGaAs layers. These dislocations might also act as centers for the domain wall nucleation in the current work. At $\boldsymbol{B}=1000 \mathrm{G}$, the magnetization direction in $\mathrm{ND}-\mathrm{F}$ is completely switched along the direction of the applied magnetic fields. However, no change in the magnetic 

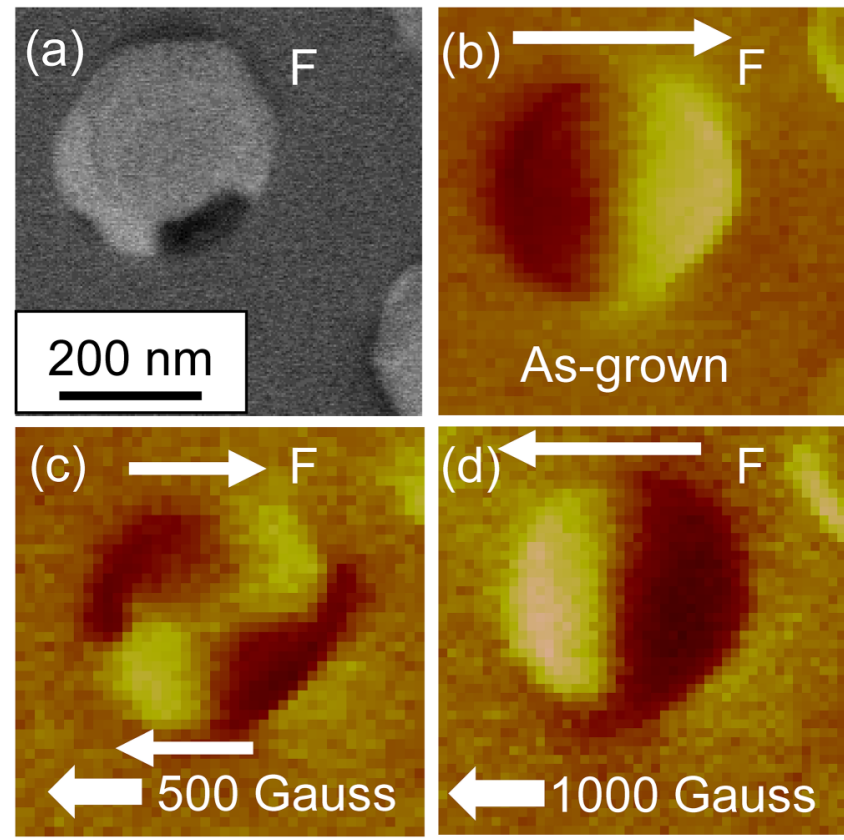

Attractive

Repulsive

FIG. 5. (a) BSE image of ND-F and the applied magnetic field dependence of the magnetic domain structure of ND-F: (b) as-grown, i.e., $\boldsymbol{B}=0$, (c) 500, and (d) $1000 \mathrm{G}$. The thick and thin white arrows indicate the directions of the applied magnetic field, $\boldsymbol{B}$, and the magnetization directions of the MnAs NDs, respectively. At $\boldsymbol{B}=500 \mathrm{G}$, a change in the domain structure to two magnetic domains from a single domain is observed. At $\boldsymbol{B}=1000 \mathrm{G}$, the magnetization direction of MnAs ND is completely reversed parallel to the direction of the applied magnetic field, $\boldsymbol{B}$.

domain structure and the magnetization direction is observed in almost all the other NDs (not shown here). Therefore, the coercive forces of NDs are supposed to be typically larger than 1000 Oe as $1 \mathrm{G}$ is approximately $1 \mathrm{Oe}$ in the air. In the 20-nm-thick MnAs films grown by molecular beam epitaxy on GaAs (001) substrates, a coercive force of approximately 400 Oe was confirmed. ${ }^{35}$ The enhancement of the coercive forces probably arises from the decrease in grain size of MnAs, as an increase of the coercive forces with decreasing grain size was reported as well for other magnetic material systems. ${ }^{36,37}$ We believe that such a feature of a relatively large coercive force enhanced in the MnAs NDs is promising for using such NDs as a pinned layer of MTJs or for our proposed planar-type magnetic logic device structures consisting of elongated MnAs nanostructure composites selectively grown on GaAs (111)B substrates. According to our previous study, ${ }^{16}$ in these nanostructure composites, the $\boldsymbol{B}$ for the magnetization reversals ranged from 750 to $1000 \mathrm{G}$.

\section{CONCLUSIONS}

We characterized the magnetic domain and domain wall structure of MnAs NDs on AlGaAs NP buffers selectively grown on $\mathrm{Si}$ (111) substrates partially covered with dielectric $\mathrm{SiO}_{2}$ thin film mask patterns using SA-MOVPE. The results on the size dependence of the magnetic domain structure in the MnAs NDs were observed by MFM and showed that a single domain is predominant in MnAs NDs with an area of approximately $3 \times 10^{4} \mathrm{~nm}^{2}$ or less. The investigations also revealed that multiple domains, in particular, a two magnetic domain structure with a $180^{\circ}$ domain wall, are predominant in NDs with an area of approximately $6 \times 10^{4} \mathrm{~nm}^{2}$ or more. In addition, in the case of NDs with multiple domains, not only Néel walls but also Bloch walls are formed, judging from the detailed analyses of the MFM images obtained. These results suggested that the magnetic domains and domain walls can be tuned by the control in the MnAs ND size.

\section{ACKNOWLEDGMENTS}

We sincerely thank Professor P. J. Klar, Dr. M. T. Elm, Professor T. Fukui, and Professor J. Motohisa for the stimulating and productive discussions we had with them, and R. Kodaira, T. Kadowaki, H. Kato, K. Morita, K. Komagata, S. Ito, H. Iguchi, T. Wakatsuki, Dr. S. Sakita, Dr. T. Sato, and Dr. Y. Kohashi for supporting the sample preparations and the characterization experiments. This work was financially supported in part by Grants-in-Aid for Scientific Research (B) and Challenging Exploratory Research (KAKENHI Grant Nos. JP17H02727 and JP16K13671) from the Japan Society for the Promotion of Science.

${ }^{1}$ H. Ohno, Science 281, 951 (1998).

${ }^{2}$ B. T. Jonker, Proc. IEEE 91, 727 (2003).

${ }^{3}$ S. Sugahara and M. Tanaka, Appl. Phys. Lett. 80, 1969 (2002).

${ }^{4}$ P. N. Hai, M. Yokoyama, S. Ohya, and M. Tanaka, Phys. E 32, 416 (2006).

${ }^{5}$ P. N. Hai, S. Ohya, M. Tanaka, S. E. Barnes, and S. Maekawa, Nature 458, 489 (2009).

${ }^{6}$ L. Thomas, S.-H. Yang, K.-S. Ryu, B. Hughes, C. Rettner, D.-S. Wang, C.-H. Tsai, K.-H. Shen, and S. S. P. Parkin, in Proceedings of the 2011 International Electron Devices Meeting (IEDM 2011) (IEEE, 2011), 24.2.1.

${ }^{7}$ A. J. Annunziata, M. C. Gaidis, L. Thomas, C. W. Chien, C. C. Hung, P. Chevalier, E. J. O’ Sullivan, J. P. Hummel, E. A. Joseph, Y. Zhu, T. Topuria, E. Delenia, P. M. Rice, S. S. P. Parkin, and W. J. Gallagher, in Proceedings of the 2011 International Electron Devices Meeting (IEDM 2011) (IEEE, 2011), 24.3.1.

${ }^{8}$ R. Takemura, T. Kawahara, K. Miura, H. Yamamoto, J. Hayakawa, N. Matsuzaki, K. Ono, M. Yamanouchi, K. Ito, H. Takahashi, S. Ikeda, H. Hasegawa, H. Matsuoka, and H. Ohno, IEEE J. Solid-State Circuits 45, 869 (2010).

${ }^{9}$ S. Peng, W. Kang, M. Wang, K. Cao, X. Zhao, L. Wang, Y. Zhang, Y. Zhang, Y. Zhou, K. L. Wang, and W. Zhao, IEEE Magn. Lett. 8, 3105805 (2017).

${ }^{10}$ K. H. Ploog, L. Däweritz, R. Engel-Herbert, and T. Hesjedal, Phys. Status Solidi B 203, 3574 (2006).

${ }^{11}$ R. W. De Blois and D. S. Rodbell, J. Appl. Phys. 34, 1101 (1963).

${ }^{12}$ S. Hara, D. Kawamura, H. Iguchi, J. Motohisa, and T. Fukui, J. Cryst. Growth 310, 2390 (2008).

${ }^{13}$ M. T. Elm and S. Hara, Adv. Mater. 26, 8079 (2014).

${ }^{14}$ S. Ito, S. Hara, T. Wakatsuki, and T. Fukui, Appl. Phys. Lett. 94, 243117 (2009).

${ }^{15}$ M. T. Elm, C. Michel, J. Stehr, D. M. Hofmann, P. J. Klar, S. Ito, S. Hara, and H.-A. Krug von Nidda, J. Appl. Phys. 107, 013701 (2010).

${ }^{16}$ S. Hara and K. Komagata, Phys. Status Solidi B 252, 1925 (2015).

${ }^{17}$ R. Horiguchi, S. Hara, M. Iida, and K. Morita, "Selective-area growth of ferromagnetic MnAs nanodisks on Si (111) substrates using multiple types of dielectric masks," J. Cryst. Growth (submitted).

${ }^{18}$ E. Sánchez, M. T. Deluigi, and G. Castellano, Microsc. Microanal. 18, 1355 (2012).

${ }^{19}$ K. Kanaya and S. Okayama, J. Phys. D 5, 43 (1972).

${ }^{20}$ J. B. Goodenough and J. A. Kafalas, Phys. Rev. 157, 389 (1967).

${ }^{21}$ F. Ishikawa, K. Koyama, K. Watanabe, and H. Wada, Jpn. J. Appl. Phys. 42, L918 (2003).

${ }^{22}$ V. M. Kaganer, B. Jenichen, F. Schippen, W. Braun, L. Däweritz, and K. H. Ploog, Phys. Rev. B 66, 045305 (2002).

${ }^{23}$ R. Engel-Herbert, T. Hesjedal, J. Mohanty, D. M. Schaadt, and K. H. Ploog, Phys. Rev. B 73, 104441 (2006). 
${ }^{24}$ R. Engel-Herbert, T. Hesjedal, D. M. Schaadt, L. Däweritz, and K. H. Ploog, Appl. Phys. Lett. 88, 052505 (2006).

${ }^{25}$ L. Däweritz, L. Wan, B. Jenichen, C. Herrmann, J. Mohanty, A. Trampert, and K. H. Ploog, J. Appl. Phys. 96, 5056 (2004).

${ }^{26}$ J. Mohanty, T. Hesjedal, T. Plake, M. Kästner, L. Däweritz, and K. H. Ploog, Appl. Phys. A 77, 739 (2003).

${ }^{27}$ Y. Nozaki, K. Matsuyama, T. Ono, and H. Miyajima, Jpn. J. Appl. Phys. 38, 6282 (1999).

${ }^{28}$ T. G. Pokhil and B. M. Moskowitz, J. Geophys. Res. 102, 22681 (1997).

${ }^{29}$ C. T. Hsieh, J. Q. Liu, and J. T. Lue, Appl. Surf. Sci. 252, 1899 (2005).
${ }^{30}$ S. Middelhoek, J. Appl. Phys. 34, 1054 (1963).

${ }^{31}$ L. Néel, Compt. Rend. Acad. Sci. 241, 533 (1955).

${ }^{32}$ H.-D. Dietze and H. Thomas, Z. Physik 163, 523 (1961).

${ }^{33}$ R. E. Behringer and R. S. Smith, J. Franklin Inst. 272, 14 (1961).

${ }^{34}$ R. Ramesh, G. Thomas, and B. M. Ma, J. Appl. Phys. 64, 6416 (1988).

${ }^{35}$ M. Tanaka, J. P. Harbison, M. C. Park, Y. S. Park, T. Shin, and G. M. Rothberg, J. Appl. Phys. 76, 6278 (1994).

${ }^{36}$ F. Phfeifer and C. Radeloff, J. Magn. Magn. Mater. 19, 190 (1980).

${ }^{37}$ G. Herzer, IEEE Trans. Magn. 25, 3327 (1989). 